\title{
Wireless instrumented klapskates for long-track speed skating
}

\author{
E. van der $\operatorname{Kruk}^{1}$ • O. den Braver ${ }^{1}$ - A. L. Schwab ${ }^{1}$ F. C. T. van der Helm ${ }^{1}$. \\ H. E. J. Veeger ${ }^{1}$
}

Published online: 17 May 2016

(c) The Author(s) 2016. This article is published with open access at Springerlink.com

\begin{abstract}
In the current project, we aim to provide speed skaters with real-time feedback on how to improve their skating performance within an individual stroke. The elite skaters and their coaches wish for a system that determines the mechanical power per stroke. The push-off force of the skater is a crucial variable in this power determination. In this study, we present the construction and calibration of a pair of wireless instrumented klapskates that can continuously and synchronously measure this push-off force in both the lateral direction and normal direction of the skate and the centre of pressure of these forces. The skate consists of a newly designed rigid bridge $(0.6 \mathrm{~kg})$, embedding two three-dimensional force sensors (Kistler 9602, Kistler Group, Winterthur, Switzerland), which fits between most individual skate shoes and Maple skate blades. The instrumented klapskates were calibrated on a tensile testing machine, where they proved to be unaffected to temperature conditions and accurate up to an RMS of $42 \mathrm{~N}$ $(\mathrm{SEM}=1 \mathrm{~N})$ in normal and up to an RMS of $27 \mathrm{~N}$ $(\mathrm{SEM}=1 \mathrm{~N})$ in lateral direction. Furthermore, the centre of pressure of these forces on the blade was determined up to a mean error of $10.1 \mathrm{~mm}(\mathrm{SD}=6.9 \mathrm{~mm})$. On-ice measurements showed the possibility of recording with both skates simultaneously and synchronously, straights as well as curves. The option to send data wirelessly and realtime to other devices makes it possible to eventually provide skaters and coaches with visual real-time feedback during practice.
\end{abstract}

E. van der Kruk

e.vanderkruk@tudelft.nl

1 Department of Biomechanical Engineering, Faculty of Mechanical Engineering, Delft University of Technology, Mekelweg 22628 CD, Delft, The Netherlands
Keywords Speed skating · Calibration · Force measurements $\cdot$ Instrumentation design $\cdot$ Real-time feedback

\section{Introduction}

Force and power production are crucial factors in any performance sport. Insight in the force pattern produced by an athlete and the related relevant velocities can help understand the technique and performance of that athlete. Ideally, we would like to provide speed skaters with realtime feedback on their skating performance within an individual stroke. The Dutch elite skaters and their coaches would like to have access to a system that determines the power per stroke, which is a familiar variable for them from cycling practices. The push-off force of the skater is a crucial variable in this power determination [1]. For power estimations, the velocity of the skater and the orientation of the skate in the global frame are a necessity. Therefore, to fulfil the wish of the skaters, accurate measurement systems that can capture the kinetic and kinematic data of a skater, and preferably send it real-time to a device for feedback (e.g., phone, tablet, smart glasses), are essential. Since the accuracy of all these measurements are of utmost importance for the quality of the feedback, we will deal with these in separate studies. In this paper, we present the construction and validation of a set of wireless instrumented klapskates that can measure the forces applied by the skater. The validation and measurements of the orientation of the skate, which will be performed with an IMU and self-designed filter, and the velocity of the skater are presented in a follow-up study.

Publication on instrumented skates started in the early 90 s of the last century. At that time, speed skaters skated on 
the conventional fixed skates, where the blade of the skate was fixed to the shoe. Two studies were published on instrumented fixed skates. The pioneers in this field aimed at measuring normal forces in speed skating on the straights and in a curve [2,3]. They constructed a right skate with three temperature compensated strain gauges that could measure in normal direction and longitudinal direction in a local coordinate system [4]; (Fig. 1). Their study on-ice friction [5] measured the longitudinal forces on the blade and demonstrated that the maximal forces in longitudinal direction were $10 \mathrm{~N}$, which is less than $1 \%$ of the force in normal direction. From these studies, we conclude that measuring this small component of force is a technical challenge, while it is negligible relative to the total force.

Yuki et al. [6] measured the normal and lateral forces in speed skating on the straight. For this study, two sensorskates were built, which consisted of strain gauges between the shoe and the blade. Their study aimed at measuring the horizontal $\left(F_{h}\right.$, Fig. 1) and vertical $\left(F_{v}\right)$ blade reaction forces for two different skating velocities, slow $(9.1 \mathrm{~m} / \mathrm{s})$ and fast $(11.5 \mathrm{~m} / \mathrm{s})$, respectively. Their study was the first to also report the centre of pressure of these static resultant forces on the skate blade (COP). Their results, based on one-skilled skater $(n=1)$, indicated that the peak lateral forces were considerable, namely between the $F_{L}=0.3-0.6 \mathrm{BW}(\mathrm{BW}=$ body weight $)$, which is equal to $25-45 \%$ of the peak normal force. Since the lateral force proved to be considerable, measuring this component

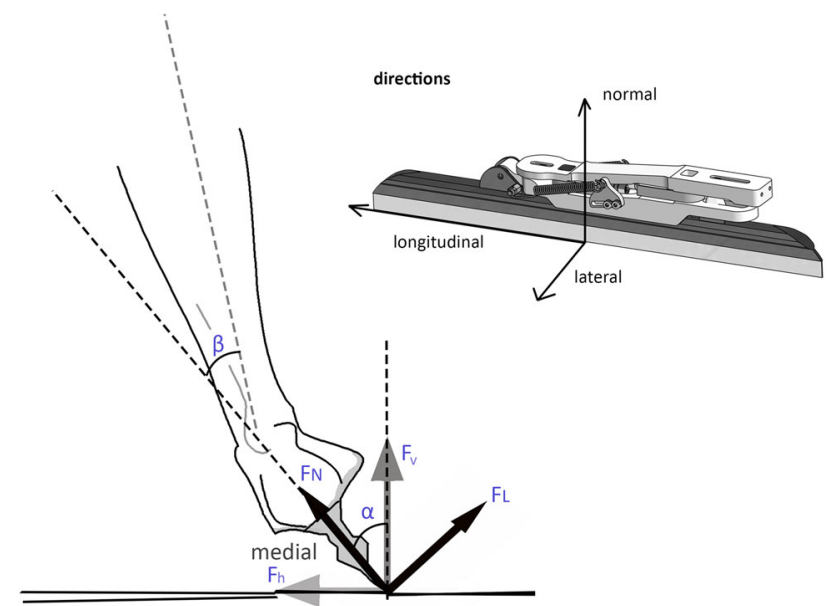

Fig. 1 Normal force $\left(F_{N}\right)$ and lateral force $\left(F_{L}\right)$ are the forces measured in the local skate coordinate system (as in $[2,4,7])$. The forces $F_{v}$ and $F_{h}$ are the vertical and horizontal components (as in [6, 8]) of the push-off forces $F_{N}$ and $F_{L} \cdot \alpha$ is the lean angle of the skate and describes the angle between the skate and the ice. $\beta$ is de angle between the shank and the skate and describes the eversion of the ankle. Given is the rear view of the right skate, the force $F_{L}$ is for both the right and the left skate negative in medial direction. The lean angle is positive, while the blade is on the medial side seems inevitable when analysing speed skating forces. Furthermore, a relationship was found between the performance of the speed skater and the COP. This makes the $\mathrm{COP}$, an esteemed variable for feedback.

In the mid-90s, fixed skates were replaced by klapskates, which incorporate a hinge between the blade and the shoe. The first constructed instrumented klapskate measured forces in normal direction, thereby again neglecting the forces in lateral direction [7]. Not long after, Yuda et al. [8] constructed a (left) instrumented klapskate that could measure in normal direction and lateral direction. The skate was equipped with two quartz voltage sensors which measured three components between the boot and the bridge of the skate. The data were logged on a computer carried on the skater's back. Their study reports on measurements in the curve with the left instrumented skate. Unfortunately, no data on the calibration or accuracy of the force measurements were reported. Drawback of the design of this instrumented klapskate was the necessity for the skaters to carry a laptop to $\log$ the data $(3.3 \mathrm{~kg})$. In addition, the sensors were placed between the skate shoe and the blade as a result of which every participant had to wear (and fit) the same (only left) skate shoe. To perform true measurements and preserve participation of many skaters, a skater needs to be able to wear his own skate shoe, and preferably blade, during measurements.

Three previous studies have described calibration procedures for instrumented skates in long-track speed skating [4-6]. In all three studies, which use static calibration with weights, the accuracy determination focused on the crosstalk between the force directions rather than the accuracy of the force directions itself. Since also the relationship between the forward velocity (performance) and the measured forces had not completely been grasped yet, literature did not provide us with an adequate benchmark for the accuracy requirement for an instrumented skate. One study has been published on the construction and calibration of an instrumented ice hockey skate, which measured the forces in normal direction and lateral direction, with strain gauges, and reported on the calibration accuracies in the separate directions [9]. Their skates were calibrated with an error of $68 \mathrm{~N}$ in normal direction and $40 \mathrm{~N}$ in lateral direction.

In this study, we present the construction and calibration of a pair of instrumented klapskates that can continuously and synchronously measure both the lateral and normal forces in a local frame and the COP of these forces. The skate is designed to fit most individual skate shoes and Maple skate blades [10]. In addition, the developed system can log data wirelessly and locally through a logger at the rear of the skate. In the design, the longitudinal force is neglected, since this is assumed to be less than $1 \%$ of the normal force. The skate is built to be part of the 
instrumentation to provide speed skaters with real-time feedback during practice on improving their performance within a single stroke.

\section{Method}

In this section, first, the design of the instrumented klapskates is described. Subsequently, the calibration set-up, the calibration routine, and calibration analyses are explained. The section closes with the description of the on-ice skating measurements.

\subsection{Instrumented klapskates}

The instrumented klapskate consists of a custom-made rigid aluminium bridge (mountable on Maple skates), with two three-dimensional piezoelectric force sensors with integrated electronics (Kistler 9602, Kistler Group, Winterthur, Switzerland) (Fig. 2). Due to the design of the bridge, the sensors are not aligned with the bridge. The voltage output of the front $\left(V_{f x}, V_{f y}, V_{f z}\right)$ and rear $\left(V_{r x}, V_{r y}\right.$, $V_{r z}$ ) sensor are logged on an SD card and sent over Bluetooth via a data logger [11]. The logger is further equipped with an accelerometer, gyroscope, and magnetometer (IMU), and logs temperature for temperature-compensation. The IMU will be used in future application for orientation measurements; these are, however, not discussed in this study. The force sensors are powered by rechargeable Li-Ion batteries. Synchronisation between the two skates is done over Bluetooth. To enable synchronisation with external measurement devices, a digital start-end

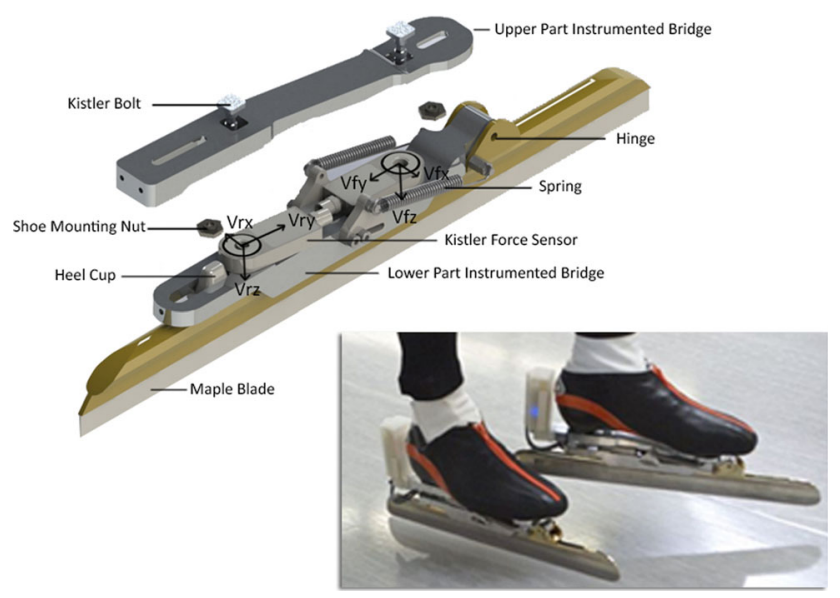

Fig. 2 Overview of the instrumented bridge and an attached blade. The lower part of the bridge is only attached to the blade via the hinge. The upper part of the bridge is attached to the lower part via the Kistler bolts under pre-stress. The skater can place its own shoe on the bridge via the shoe mounting nuts. The small white box near the heel is the recording and transmission unit for the measured data pulse can be logged. The weight of the instrumented bridge and electronics is $600 \mathrm{~g}$. The instrumented bridge replaces the normal bridge in a klapskate. It can be attached to any Maple blade via the hinge mechanism, and any skate shoe can be placed on it. The instrumented bridge does not increase the height of the skate (Fig. 3).

\subsection{Calibration}

The calibration of the instrumented skates was performed using a tensile testing machine (Zwick Z100, Zwick Roell, Ulm, Germany, principal accuracy $1 \mathrm{~N}$ ). The aim of the calibration was to calibrate the forces on the instrumented skates in normal direction and lateral direction and the centre of pressure of these resultant forces on the blade (COP). Since it was uncertain whether the measurements of the skate were influenced by the low environmental temperature on the ice rink, ranging between -5 to $0{ }^{\circ} \mathrm{C}$ on the ice and 0 to $5{ }^{\circ} \mathrm{C}$ just above the ice, first, the temperature dependency was determined.

The tensile testing machine was placed in a climatecontrolled chamber to regulate the temperature. Each skate was positioned blade up in four different positions (P1-P4) on the fixed lower head of the testing machine. The reference force $F_{L C}$, exerted by the movable upper head of the machine $(\varnothing 50 \mathrm{~mm})$, was applied directly to the blade via a constant displacement, up to a maximum force, and

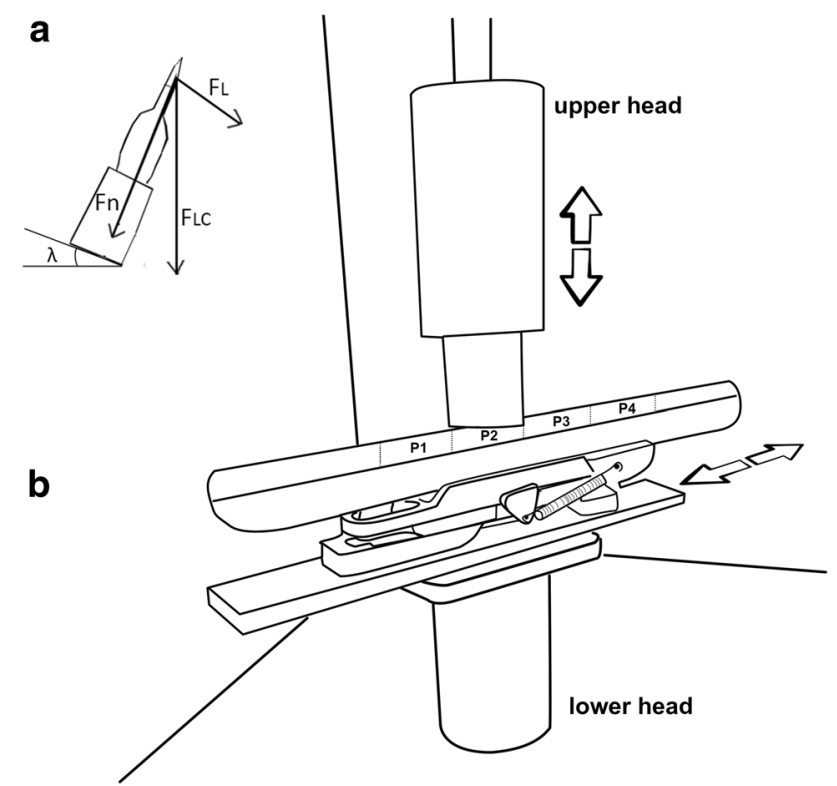

Fig. 3 Calibration Set-up; a the tensile strength machine exerts the force $F_{L C}$, due to the tilting angle $\lambda$ under which the skate is positioned, this results in a force in normal direction and lateral direction. b The upper head moves down to exert force on the blade of the instrumented skate. The skate can be placed in five different positions $(P 1-P 5)$ to calibrate the COP and can be placed under an angle $\lambda$ (see Fig. 2) to calibrate the normal and lateral forces 
released by the same constant displacement. A wedge was designed to place the skate under five tilting angles $\left(\lambda_{t a}\right)$ with $\lambda_{t a}=-7^{\circ}, 7^{\circ}, 0^{\circ},-20^{\circ}, 20^{\circ}$, to distribute the applied force $F_{L C}$ into a normal $\left(F_{N}\right)$ force and a lateral $\left(F_{L}\right)$ force on the skate.

\subsubsection{Temperature}

The temperature dependency test was conducted under four different temperatures $\left(-5,0,5\right.$ and $\left.19{ }^{\circ} \mathrm{C}\right)$ at position $\mathrm{P} 2$, by applying a rising force up to 1100 N. From each condition, 12 samples were taken (every $100 \mathrm{~N}$ ) and the combined output voltages $V_{f z}+V_{r z}$ were compared between temperature conditions. Results showed that the output voltages were uncorrelated to the temperature conditions $(R=-0.015, \quad p=0.89$ for left skate and $R=-0.0037, p=0.9712$ for the right skate). Therefore, the remaining calibration could be performed at room temperature $\left(19^{\circ} \mathrm{C}\right)$.

\subsubsection{Normal force and lateral force}

The calibration in normal direction and lateral direction was performed at room temperature $\left(19^{\circ} \mathrm{C}\right)$, in four different positions (P1-P4) under the five tilting conditions $\lambda_{-7^{\circ}}, \lambda_{7^{\circ}}, \lambda_{0^{\circ}}, \lambda_{-20^{\circ}}$, and $\lambda_{-20^{\circ}}$. At each position, under each condition, 12 samples were taken from the force data, equally divided from zero up to the maximum measured force. The maximum forces differed between conditions due to the applied tilting angle, but ranged from $1600 \mathrm{~N}$ to $2500 \mathrm{~N}$ in normal direction and from $-610 \mathrm{~N}$ up to $610 \mathrm{~N}$ in lateral direction.

Calibration was done using a forced entry regression analysis with a second-order fit in both the normal direction and lateral direction. This second-order fit was believed to be necessary due to non-linearity in the material deformations of the bridge, the heel cup and the spring and to intercept any differences in pre-stress of the sensors. The characteristics of the heel cup and the spring mainly influence the force transition in lateral direction and its influence varies with the position of the applied forces in lateral direction. Therefore, the ratio RV was introduced as a measure of this position:

$R V=\frac{V_{f z}-V_{r z}}{V_{f z}+V_{r z}}$

This ratio was introduced as an input into the lateral regression, to improve the lateral force estimation. Overall, input for the second-order regression analysis in normal direction was the voltages measured in the vertical plane $\left(V_{f z}\right.$ and $V_{r z}$ ). Input to the second-order model in lateral direction was the voltages measured in the lateral direction $\left(V_{f x}\right.$ and $\left.V_{r x}\right)$ and the ratio $\mathrm{RV}$.

\subsubsection{Centre of pressure}

The COP is the position on the skate blade, where the resultant force acts on. Input for a forced linear regression for determination of the COP was the ratio RV:

$\mathrm{COP}=c_{0}+c_{1} \cdot \mathrm{RV}$

where $c_{0}$ and $c_{1}$ are the variables to be determined by regression. The assumption was made that the applied force acts at the centre of the moving upper head, neglecting the curving of the blade, and that the point of application at the blade is equal for the normal and lateral applied forces.

\subsection{Measurements on the ice rink}

To demonstrate the practical use of the measurement system, data were collected in 2015 on the indoor ice rink of Thialf, which is located at Heerenveen in The Netherlands. Seven Dutch elite speed skaters $(5 \hat{\jmath}, 2 \circ ; 22 \pm 1.1$ years; $77.3 \pm 6.8 \mathrm{~kg}$; PR at $1000 \mathrm{~m}$ : ð̋1.10-1.12 s, ㅇ 1.16-1.18 s) were equipped with two instrumented skates, on which their own skating shoes were positioned. The average velocity over a straight part or a curve was measured by a local position measurement system [12]. Skaters familiarized themselves with the equipment before the start of the test. The test was divided into three parts, each at a different velocity, which each consisted of skating three laps at a constant velocity. We employed the data at $10.3 \mathrm{~m} / \mathrm{s}$ for the current paper. The push-off forces were normalized to ratio of total body weight and equipment (BW). The onset of a stroke was defined as the time at which the normal force attained $100 \mathrm{~N}$ [2], the end of the stroke the time at which the normal force fell back to $100 \mathrm{~N}$. The time is normalized in units of stroke time.

\subsection{Statistics}

The statistics applied in this paper are based on three strokes per participant, per side. Therefore, 21 strokes for each skate (left and right). To establish any differences in peak forces or mean COP between left and right, a paired sample $t$ test was performed, in which the mean value of three strokes was used as an input for each participant.

\section{Results}

\subsection{Calibration results}

Calibration in normal direction with a second-order regression equation resulted in correlations of $R^{2}=0.995$ and $R^{2}=0.997$ for the left and the right skate, respectively, with a root-mean-square error (RMS) of $42 \mathrm{~N}$ 
$(\mathrm{SEM}=1 \mathrm{~N})$ and $38 \mathrm{~N}(\mathrm{SEM}=1 \mathrm{~N})$ (Fig. 4) ( $\mathrm{SEM}$ is the standard error of the mean). Calibration in lateral direction performed with a second-order regression, incorporated the factor RV. The correlation for the lateral direction yielded $R^{2}=0.991$ for both skates with the corresponding RMS errors of $25 \mathrm{~N}(\mathrm{SEM}=1 \mathrm{~N})$ and
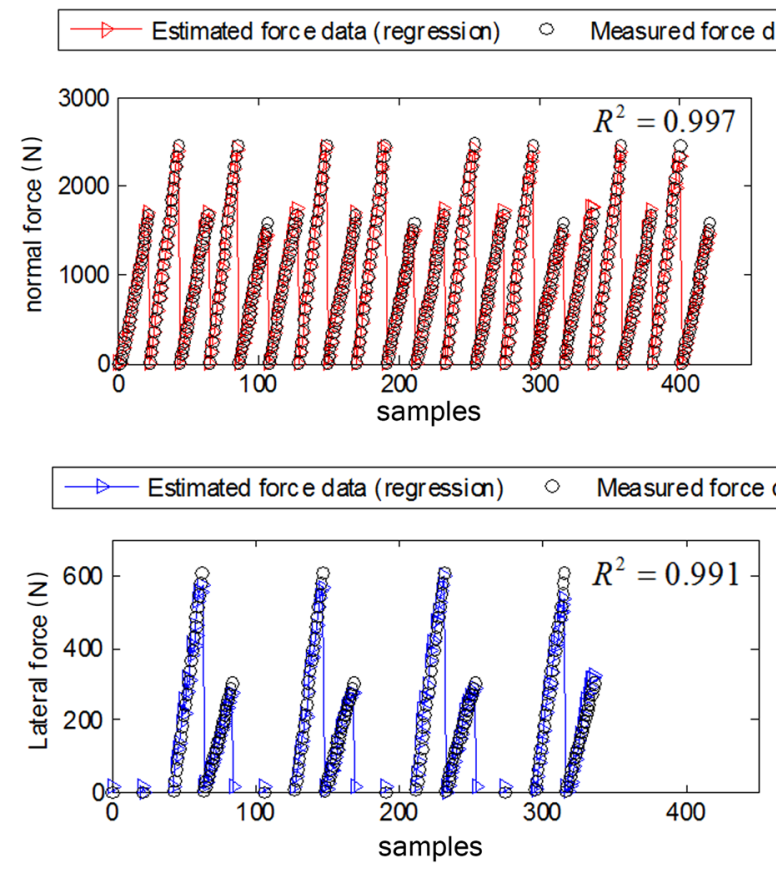

Fig. 4 Forces estimated by the right instrumented skate with the second-order regression functions and the forces measured by the tensile test machine for each sample. The upper graph shows the results for the normal direction, the lower graph the results for the lateral direction. Given are the regression coefficients of the fit
$27 \mathrm{~N}(\mathrm{SEM}=1 \mathrm{~N})$ for, respectively, the left skate and the right skate. The remaining error of the fit proved to be random. The absolute mean error of the centre of pressure estimation, based on linear regression (Eq. 2), is $8.5 \mathrm{~mm}$ $(\mathrm{SD}=6.4 \mathrm{~mm})$ for the left skate and $10.1 \mathrm{~mm}$ $(\mathrm{SD}=6.9 \mathrm{~mm})$ for the right skate.

\subsection{Measurements on the rink}

Figure 5 shows an example of the force registration for two straights and a curve. The curve is characterized by a higher stroke frequency and lower normal forces compared to the straight parts. Furthermore, the lateral forces of the left skate change direction when entering the curve, since the skater changes the push-off for this skate from the medial side to the lateral side of the blade. Except for normal forces in the curve, all other forces show a clear peak at the end of the stroke. Figure 6 presents the normalized forces (mean \pm SD of seven participants, each three strokes) with a velocity of $10.3( \pm 0.6) \mathrm{m} / \mathrm{s}$ for the straight part. The mean peak values of the normalized normal forces at the straight part were $F_{N}=1.35( \pm 0.09) \mathrm{BW}$ and $F_{N}=1.38( \pm 0.08)$ BW for the left and right strokes, respectively. The mean maximum normal forces in the curve (Fig. 7) go up to $F_{N}=1.30( \pm 0.07) \mathrm{BW}$ and $F_{N}=1.32( \pm 0.09) \mathrm{BW}$ for the left and the right strokes, respectively. The lateral forces showed mean peak values at the straight part of $F_{L}=0.74$ $( \pm 0.14) \mathrm{BW}$ and $F_{L}=0.79( \pm 0.25) \mathrm{BW}$ for the left and right skates. There is no significant difference between left and right for any of these maximum forces $(p>0.1)$; however, the lateral forces in the curve do have mean peak values that differ between sides $(p<0.00)$, with $F_{L}=0.35$
Fig. 5 Example of a force registration of a female elite skater $(65 \mathrm{~kg})$ on two straights and a curve part at $10 \mathrm{~m} / \mathrm{s}$. The lateral forces are positive for both the left skate and the right skate at the straight parts. In the curve, the force on the left skate is applied on the medial side of the blade

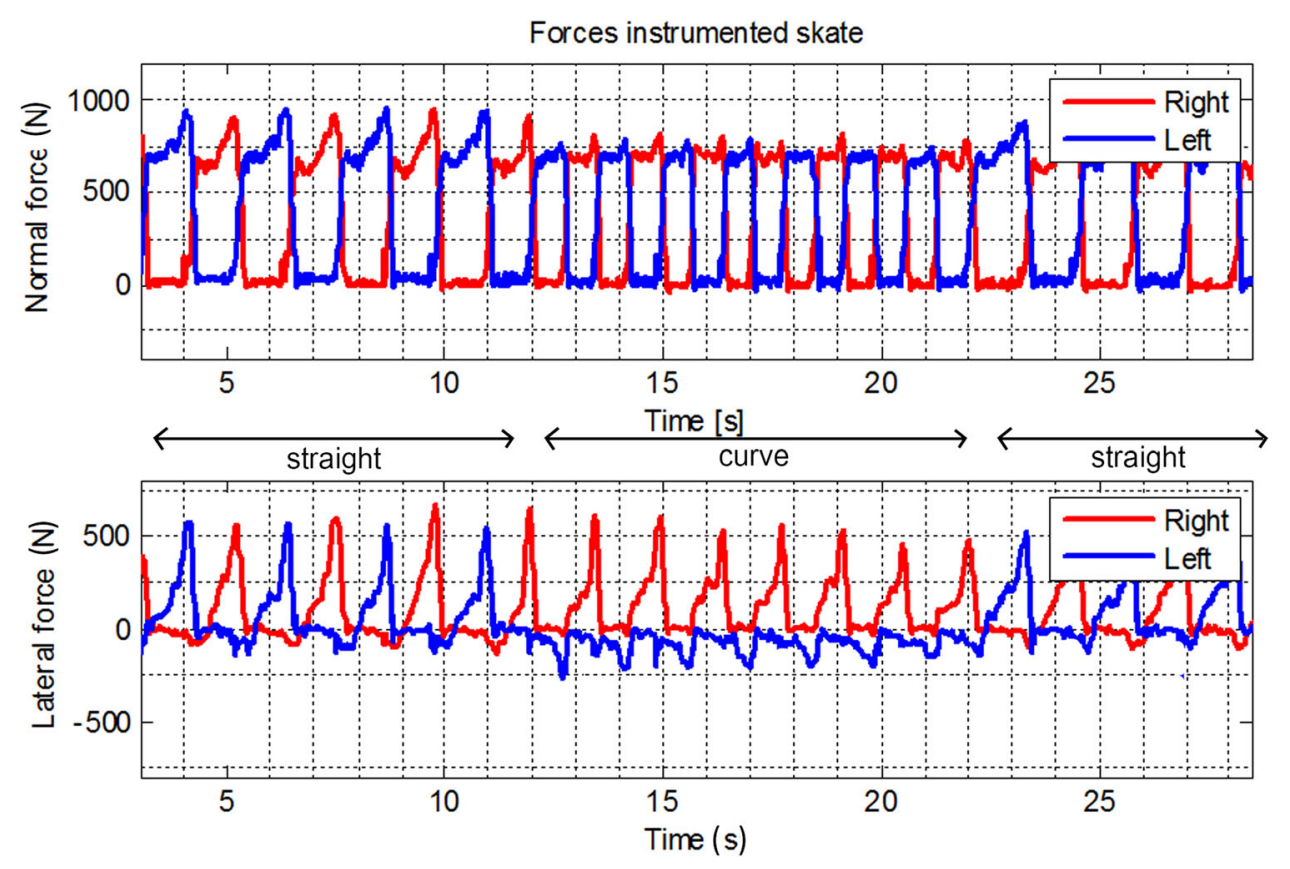


Fig. 6 Mean and standard deviation for the normal and lateral forces for seven elite speed skaters measured at the straight. The forces are presented per stroke from each skater three strokes were incorporated. The forces are normalized to body weight and the stroke is normalized to stroke time

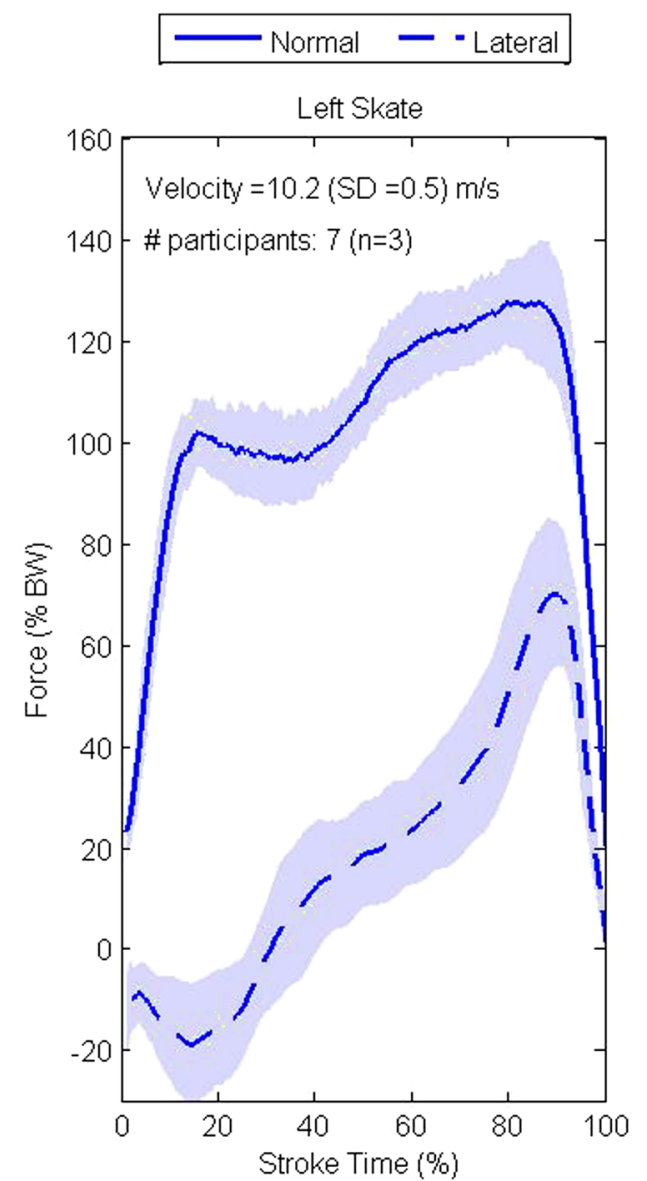

$( \pm 0.09) \mathrm{BW}$ for the left stroke and $F_{L}=0.73( \pm 0.25) \mathrm{BW}$ for the right stroke.

The mean COP (Fig. 8) showed no difference between left and right on the straight part $(p=0.58)$, while in the curve, there is a clear difference. In the curve, the COP moves faster toward the front of the blade compared to the straight, which is most evident in the left skate. The timing of the peak forces is indicated by the vertical grey bar in the figure. Except for the left COP in the curve, all peak forces occur, while the COP moves to the front of the blade. For the left skate in the curve, the COP already shifts towards the hinge (at $0 \mathrm{~mm}$ ) before the occurrence of peak forces, and levels while the peak forces occur.

\section{Discussion}

\subsection{Construction and calibration}

In this paper, we have described the construction and calibration of two wireless instrumented klapskates that can measure the lateral and normal forces during high-speed skating with an accuracy of RMS of $42 \mathrm{~N}(\mathrm{SEM}=1 \mathrm{~N})$ in normal and up to an RMS of $27 \mathrm{~N}(\mathrm{SEM}=1 \mathrm{~N})$ in lateral direction. As mentioned in the introduction, we cannot determine yet whether the found accuracy will be sufficient for the purpose of providing elite speed skaters with adequate feedback on improving their performance, since the relation between the forward velocity (performance) and the applied forces is yet to be discovered. This relationship can, however, be established with for instance a dynamic model of skating that describes the complete path from push-off force to forward velocity. The current skate can be of use in validating such a model [13].

Apart from crosstalk, previous papers on the calibration of instrumented skates for speed skating did not give an accuracy indication for separate force directions. The study on the construction and calibration of an instrumented ice hockey skate did [9]. The RMS errors of the instrumented klapskates are similar to those of the ice hockey skates, with an RMS in normal direction of $42 \mathrm{~N}$ for the klapskates versus $68 \mathrm{~N}$ for the ice hockey skates and an RMS in lateral direction of $27 \mathrm{~N}$ for the klapskates and $40 \mathrm{~N}$ for the ice hockey skates.

The skates were calibrated in two directions, lateral and normal, while the sensors are capable of measuring in three directions. We intentionally chose to neglect the longitudinal direction, due to the relatively small forces acting in 
Fig. 7 Mean and standard deviation for the normal and lateral forces for seven elite speed skaters measured at the curve. The forces are presented per stroke from each skater three strokes were incorporated. The forces are normalized to body weight and the stroke is normalized to stroke time
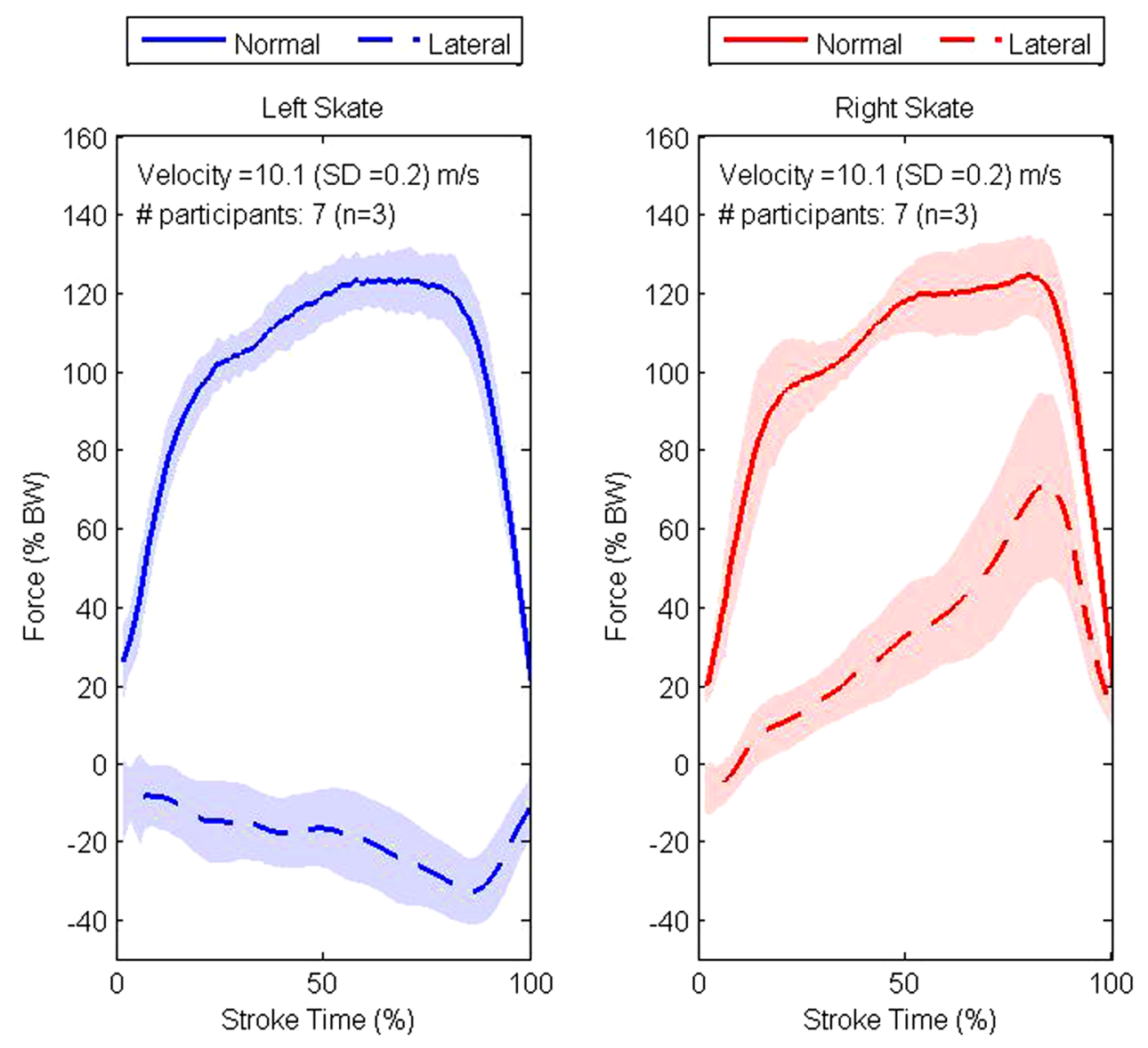

Fig. 8 Centre of Pressure of the resultant force $(\mathrm{COP})$ for the straight part and the curve. Given are the mean and standard deviation of the measured COP of 7 participants, each three strokes. The grey area indicates the occurrence of peak forces. The hinge is located at $0 \mathrm{~mm}$, the damper at $-205 \mathrm{~mm}$, and the centre of the heel cup at $-220 \mathrm{~mm}$

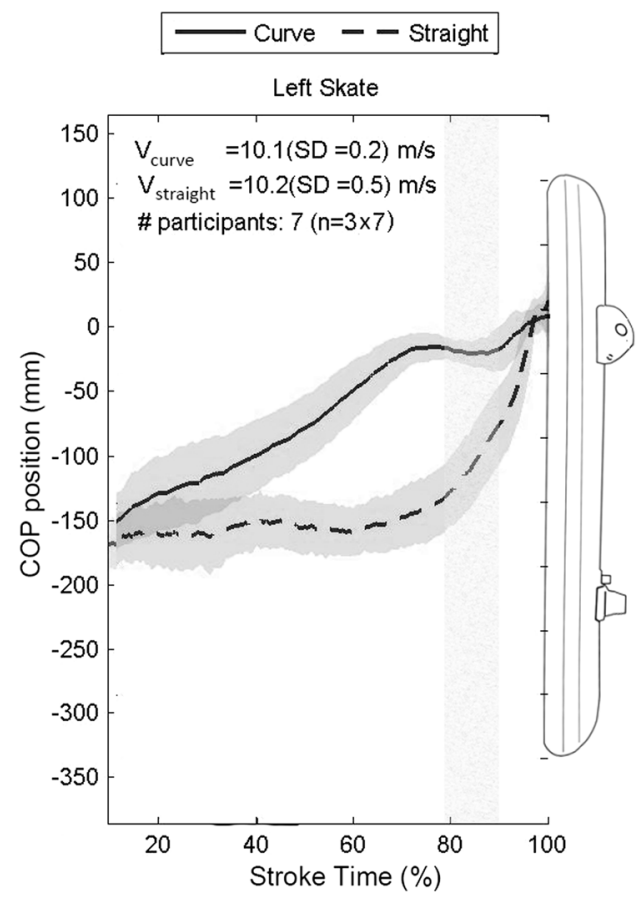


this direction. Considering the crosstalk of $\approx 3 \%$ between the vertical and transverse plane of the applied sensors, as reported by the manufacturer, it will be impossible to observe ice friction forces lower than $1 \%$ of the body weight with the current design. If in future research, we do want to determine these forces, a construction needs to be made, whereby the normal forces are completely decoupled from the transverse plane, to avoid crosstalk. With the current design, this will not be feasible.

The aim to build wireless instrumented klapskates was accomplished in this paper. Although the instrumented skates function as a klapskate, the skate was only calibrated, while it was closed. As soon as the skate opens, the force measurements are, therefore, no longer reliable. Previous studies indicate that the opening of the skate happens at the very last part of the stroke $(50 \mathrm{~ms}$ before lifting the skate) and that the forces are small in this time span [7]. As a consequence, we expect this not to be a major problem for the power determination. The benefit of the hinge mechanism in the system is to preserve the skating movement of speed skaters during testing. The presented instrumented skate has high resemblance to a standard klapskate. Still the influence of the added weight of the instrumented bridge on the skaters technique should not be disregarded in future analyses. A follow-up research, in which the skating technique, while skating on the instrumented skate, is compared to the technique employed, while skating on common klapskates, can identify such specific differences.

\subsection{Practical results}

The recorded normal peak forces at the straight partially agree with previous studies, where normal peaks between the $1.2-1.5 \mathrm{BW}$ at $9-11.5 \mathrm{~m} / \mathrm{s}$ on a conventional skate were reported $[2,6]$. In line with the current study, previous studies report that no clear normal peak forces were found in the curve. The lateral forces measured in the curve are higher than the reported lateral forces of Yuki et al. [6], who found lateral forces on the straight part of $0.4 \mathrm{BW}$ for both sides, and in the curve $0.3 \mathrm{BW}$ for the left and $0.6 \mathrm{BW}$ for the right skate, for one typical example. Direct comparison is, however, not feasible, since the velocity and the skill level of this skater will influence these values, and these were unfortunately not mentioned in his study. Nonetheless, both studies showed that the lateral force is a major component in the push-off force. The lateral forces are related to the lean angle of the skate and the eversion of the skate, which causes the foot and the lower leg to be unaligned. Whether the lateral force is of benefit or counterproductive to the forward motion of the skater, depends on the orientation of the skate, determined by both the lean and steer angles. The lean angle of the skate has a direct effect on the distribution of a push force over the local vertical and transverse components, while the steer angle determines the direction of push-off in the horizontal plane (forward or sideways).

\subsection{Centre of pressure}

The centre of pressure indicates where the resultant measured forces act on the skate blade. On the straight part, the centre of pressure of the resultant force levelled at about $20-70 \mathrm{~mm}$ ahead of the heel cup, for the first $80 \%$ of the stroke. Then, the COP shifted towards the hinge of the skate, in which time the peak forces occurred, followed by the opening of the skate. Since the hinge is taken as origin, the skate would open when the COP becomes positive if it was not for a spring acting on the hinge, which makes it difficult to interpret the exact opening of the klapskate. The curve showed different COP patterns for left and right. Both lack the levelled phase at the start of the stroke, since the COP continuously shifts towards the front. The left skate has a faster shift and levels at the end of the stroke, while the peak forces occur. This pattern arises from the fact that the skater has to cross his right leg over his left in the curve. Therefore, the skater is forced to move his left leg backwards faster, whereby his COP quickly shifts towards the point of his blade.

\subsection{Real-time feedback}

Measured outputs were sent wirelessly and real-time over Bluetooth to a phone carried by the speed skater during the test. It is possible to link the phone via wifi or a mobile network to a tablet or smart glasses, to provide both coach and skater with real-time visual feedback on the force level and COP during training. Eventually, the forces will be combined with kinematic measurement systems, to provide feedback on power per stroke. During this experiment, the possibility of synchronising the skates with other measurement equipment via a digital end-start pulse proved to be easy.

\section{Conclusion}

Two wireless force measuring instrumented klapskates were constructed and calibrated on a tensile testing machine, where they proved to be unaffected by temperature conditions and accurate up to an RMS of $42 \mathrm{~N}$ $(\mathrm{SEM}=1 \mathrm{~N})$ in normal direction and $27 \mathrm{~N}(\mathrm{SEM}=1 \mathrm{~N})$ in lateral direction. Furthermore, the centre of pressure of 
these forces on the blade was determined up to a mean error of $10.1 \mathrm{~mm}(\mathrm{SD}=6.9 \mathrm{~mm})$. The design of the skate allows a skater to attach his own shoe and Maple blade to the bridge. On-ice measurements showed the possibility of recording with both skates simultaneously and synchronously both straights and curves, and the capability of the system to send data wirelessly and real-time to other devices, which makes it possible to eventually provide skaters and coaches with visual real-time feedback during practice. With the construction of these instrumented klapskates, we are one step closer to fulfilling the wish of the Dutch elite skaters and their coaches for a system determining the mechanical power per stroke.

Acknowledgments The authors gratefully acknowledge the mechanical and electronics engineers N. Linskens and G. F. Liqui Lung for their support, while producing the instrumented skates and express their gratitude to A. C. Riemslag for the technical support during the calibration experiment. This study was supported by the NWO-STW under Grant 12870.

\section{Compliance with ethical standards}

\section{Conflict of interest None.}

Open Access This article is distributed under the terms of the Creative Commons Attribution 4.0 International License (http://crea tivecommons.org/licenses/by/4.0/), which permits unrestricted use, distribution, and reproduction in any medium, provided you give appropriate credit to the original author(s) and the source, provide a link to the Creative Commons license, and indicate if changes were made.

\section{References}

1. Van Ingen Schenau GJ, Cavanagh PR (1990) Power equations in endurance sports. J Biomech 23(9):865-881

2. de Koning JJ, de Boer RW, de Groot G, van Ingen Schenau GJ (1987) Push-off force in speed skating. Int J Sport Biomech 3:103-109

3. Van Ingen Schenau GJ (1981) A power balance applied to speed skating. Vrije Universiteit, Amsterdam

4. Jobse H, Schuurhof R, Cserep F, Schreurs AW, De Koning JJ (1990) Measurement of push-off force and ice friction during speed skating. Int J Sport Biomech 6:92-100

5. De Koning JJ, De Groot G, Van Ingen Schenau GJ (1992) Ice friction during speed skating. J Biomech 25(6):565-571

6. Yuki M, Ae M, Fujii N (1996) Blade reaction forces in speed skating. Soc Biomech 13:41-51

7. Houdijk H, de Koning JJ, de Groot G, Bobbert MF, Van Ingen Schenau GJ (1999) Push-off mechanics in speed skating with conventional skates and klapskates. Med Sci Sports Exerc 32(3):635-641

8. Yuda J, Yuki M, Aoyanagi T, Fujii N, Ae M (2004) Changes in blade reaction forces in speed skating the curve. Int J Sport Heal Sci 2(1996): 195-204

9. Stidwill TJ, Turcotte RA, Dixon P, Pearsall DJ (2010) Force transducer system for measurement of ice hockey skating force. In: International Sports Engineering Association 2010

10. Maple, "Maple," http://www.mapleskate.com. Accessed 01 Sept 2015

11. Shimmer3, "Shimmer," 2015. [Online]. Available: http://www. shimmersensing.com. Accessed 01 Sept 2015

12. LPM, "LPM," http://www.inmotio.eu/en-GB/20/lpm-technology. html. Accessed 01 Sept 2015

13. van der Kruk E, Veeger HEJ, van der Helm FCT, Schwab AL (2015) Two Body Dynamic Model for Speed Skating Driven by the Skaters Leg Extension. In: icSports conference 2015 